\title{
Grado de persistencia morfológica de las formas estuariales del Maule (Chile Central) desde 1944 hasta 2011
}

\author{
Victoria Venegas¹, vagrisss@gmail.com; María-Victoria Soto² ${ }^{2}$ José F. Araya-Vergara² $^{2}$
}

\section{RESUMEN}

Se estudia el grado de persistencia de las formas estuariales del Maule, considerando las diferencias de su estructura interna y la teoría sistematica acumulada. Para el reconocimiento de formas se usó cartografías históricas y fotointerpretación cubriendo un lapso de 67 años, terminado en 2011. El trabajo de terreno consistió en la descripción de bancos, flechas y barras y su estado en el último año del período observado. La generalizada persistencia de los bancos del delta estuarial, sin cambios notables de forma y de tamaño, indica que el balance entre erosión y progradación fluvial opera bajo un régimen de retroalimentación negativa, sin cambios fundamentales en el régimen fluvial. En este sentido, se puede admitir que esta forma está controlada por un mecanismo de estabilidad fluvial, funcionando como delta estuarial de dominio fluvial. En contraste, la falta de las flechas que controlan la laguna estuarial y de las barras de la zona marina induce a concluir que estas formas son dominadas por oleaje de alta energía. Desde el punto de vista morfodinámico, las comparaciones entre el caso del Maule y otros deben hacerse separando las zonas de delta estuarial, laguna estuarial y marina.

Palabras clave: Delta estuarial, morfodinámica estuarial, cambios estuariales, persistencia geomorfológica.

\section{Degree of morphological persistence of the Maule estuarine landforms (Chile Central) since 1944 up to 2011}

\begin{abstract}
This work explores the degree of persistence of the estuarine forms of Maule, considering the differences in their internal structure and the cumulative systematic theory. For the recognition of landforms, were used historical maps and photo interpretation covering a lapse of 67 years, completed in during 2011. The fieldwork consisted in the description of banks, spits, bars, and their state in the last year of the period of observation. The widespread persistence of the banks of the estuarine delta, without notable changes of shape and size, indicates that the balance between erosion and fluvial progradation operates under a regime of negative feedback, without fundamental changes in the fluvial regime. In this sense, it appears that a mechanism of river stability regulates this landform, being a river dominated estuarine delta. In contrast, the lack of persistence of the spits that control the estuarine lagoon and the bars of the marine zone leads to conclude that these zones of the estuarine system are response to a high wave energy, being wave-dominated parts in the estuarine system. Therefore, the comparisons between the system of Maule and others should be separating the estuarine delta, estuarine lagoon and marine zone.
\end{abstract}

Keywords: Estuarine delta, estuarine morphodynamics, estuarine changes, geomorphological persistence

Recibido el 10 de marzo de 2014, aceptado el 10 de julio de 2015.

\footnotetext{
1 Memorista Departamento de Geografía, Universidad de Chile, Portugal 084, Santiago, Chile, Santiago, Chile.

2 Departamento de Geografía, Universidad de Chile, Santiago, Chile.
} 


\section{INTRODUCCIÓN}

Durante fines del siglo XIX y principios de siglo XX fueron dibujadas las principales formas insertas en el estuario del Maule. De su comparación, se pudo extraer la primera noción de cambios en bancos y barras del sistema (LÉVÉQUÉ 1877; CORDEMOY 1893; Fig. 4). Posteriormente, POMAR (1962) deduce un relleno creciente de sedimentos en el estuario, a juzgar por las dificultades para la entrada de barcos desde el mar. Este autor parece ubicar el comienzo de este proceso en el siglo XIX. Sin embargo, la falta de registros anteriores no admite deducir para este lapso un crecimiento sostenido de las formas deposicionales en el estuario. Más modernamente, cambios presentes notorios en la forma de los bancos y profundidades observados en el interior de la ría (DIRECCIÓN DE OBRAS PORTUARIAS \& LABORATOIRE CENTRALE D'HYDRAULIQUE DE FRANCE, 1960-1) sólo admiten deducir un cierto balance entre sedimentación y erosión estuarial, pero no una tendencia determinada que indique el predominio de uno de estos dos procesos..

Las primeras determinaciones geomorfológicas modernas sobre estos sistemas fueron presentadas por ARAYAVERGARA (1981, 1982 y 1985), En estos trabajos se encuentra que los sistemas de delta estuarial de Chile Central tienen una estructura común. Para ellos se usó por primera vez la expresión delta en ría, la que fue reemplazada posteriormente por delta estuarial. En un importante trabajo sobre tipificación de áreas de desembocaduira fluvial (MIKHAILOV \& GORIN, 2012) se propone la categoría estuarial-deltaico (estuarine-deltaic), definida como delta dentro de un estuario y que puede considerarse equivalente a delta estuarial. Esta forma pasa a ser considerada como parte esencial de un sistema compuesto por tres zonas: meandros estuariales, delta estuarial y laguna estuarial (ARAYAVERGARA 1981). Sobre esta base, se estableció un dominio morfoclimático para
Chile Central, que incluye el delta estuarial como forma clave, indicada como respuesta fluvio-marina al ambiente de alta energía del oleaje, característico de esta costa (ARAYA-VERGARA 1982 y 1985).

Estas experiencias han sustentado la base teórica para investigaciones más recientes en Chile Central sobre morfodinámica y evolutión estuarial, en los estuarios del río Aconcagua (CORTEZ 2002; MARTINEZ \& CORTEZ 2008) y del río Maipo (ARRIAGADA 2005).

Teniendo en cuenta este estado del asunto, el propósito del presente trabajo es estudiar el grado de persistencia de las formas estuariales del Maule, considerando las diferencias de su estructura interna y la teoría sistematica acumulada.

\section{MATERIALES Y MÉTODOS}

\section{Material estudiado}

La elección del sistema estuarial estudiado se debe a que representa un dominio de costa tectónica, abrasional, acumulativa y una forma clave consistente en delta estuarial (ARAYA-VERGARA 2007).

La costa en que se emplaza el estuario del Maule recibe esencialmente la influencia de un río de régimen pluvial y nival (Tabla 3 ), interactuando con un régimen micromareal y oleaje con mar de fondo de alta energía, hechos ya expuestos en ARAYA-VERGARA (1981). La relación entre el ambiente de oleaje y la masa sedimentaria litoral se traduce en una generalizada deriva a lo largo de la línea litoral y en dirección S-N (ARAYA-VERGARA 2007; DAVIES 1977; DAVIES 1982). Las formas asociadas a este estuario fueron analizadas en sus últimos 6 $\mathrm{km}$, en donde se identifican el delta estuarial, la laguna estuarial y la zona marina (Fig. 1). Todo el sistema está inserto en una ría con fuertes vertientes, modelada en el sistema de terrazas marinas (Fig. 2B). 


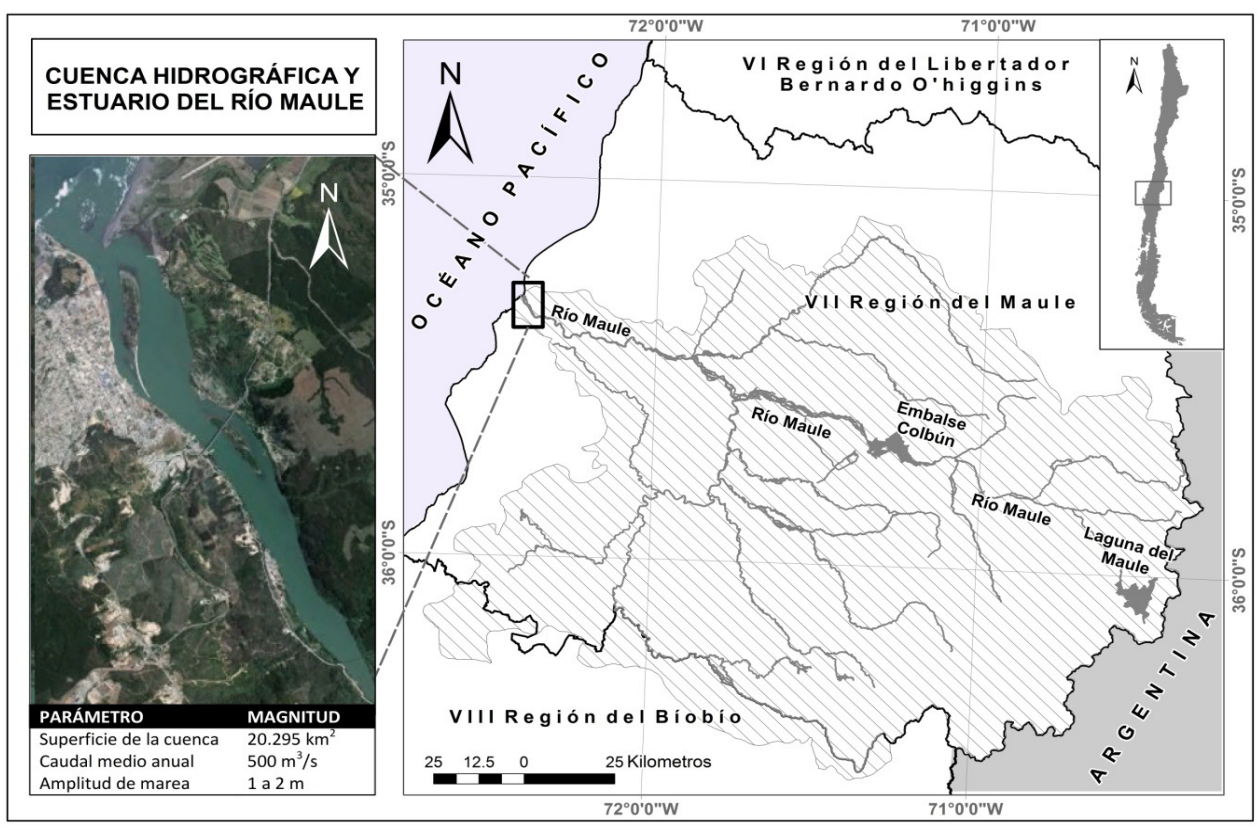

Fig. 1. Área de estudio y la cuenca asociada.

Fig. 1. Catchment and study area
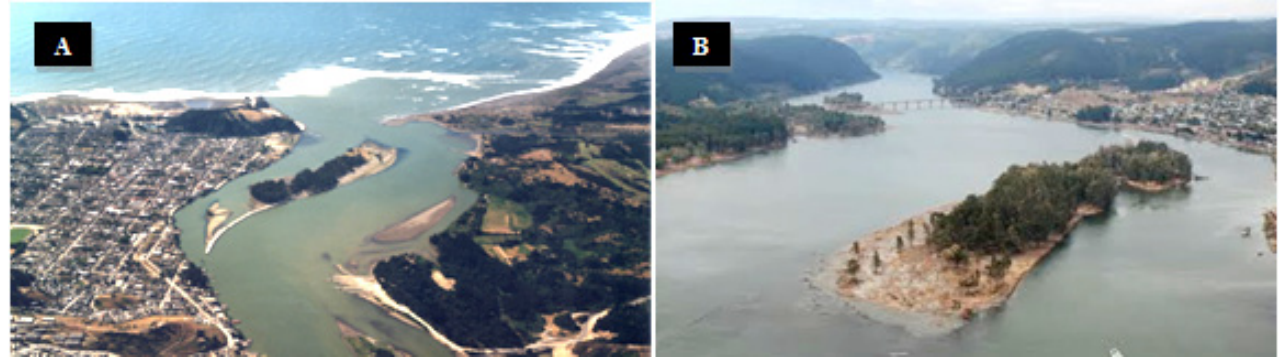

Fig. 2. Desembocadura del estuario Maule. A) Vista panorámica del sistema estuarial de Constitución, año 1999. Fuente: Instituto Hidráulico Nacional. B) Estuario del río Maule en dirección a la ría de Constitución 03 de Marzo 2010. Fuente: G. Latorre.

Fig. 2. The mouth of Maule estuary. A) Panoramic view of estuarine system of Constitución, 1999. Source: Instituto Hidráulico Nacional. B) Maule estuary looking toward Costitución ria.

Los individuos morfológicos reconocidos, fueron clasificados según la zonación de ARAYA-VERGARA (1981) para los estuarios de Chile central Fig. 3 


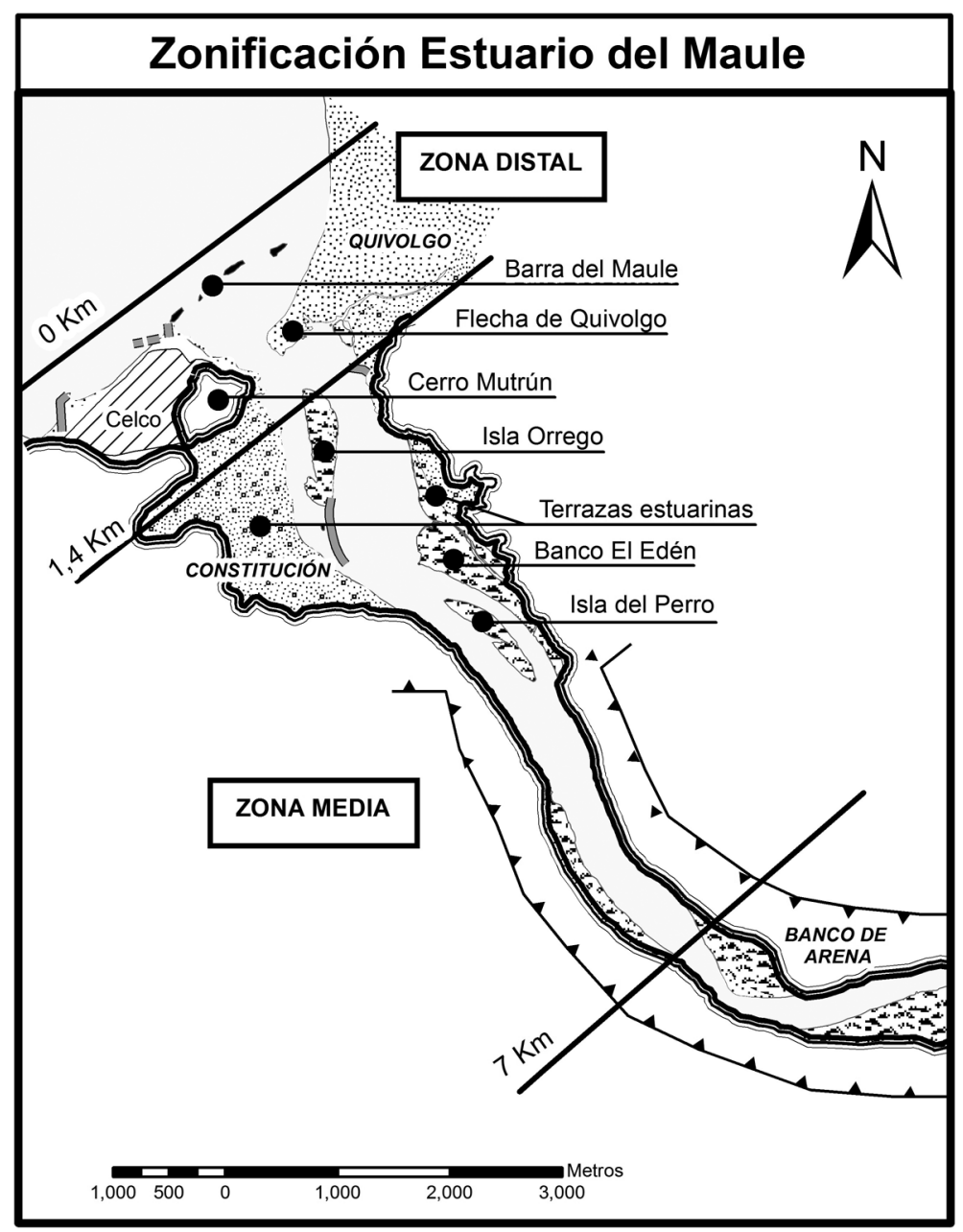

Fig. 3. Zonificación del estuario del río Maule.

Fig 3. Zoning of Maule estuary.

\section{Procedimientos}

El reconocimiento de las formas esenciales del estuario del río Maule tuvo como sustento el estudio de ARAYA-VERGARA (1981), junto con cartografías históricas, y fue realizado principalmente mediante fotointerpretación y trabajo de terreno.

Los antecedentes morfológicos históricos del estuario del río Maule, fueron extraídos a partir del análisis de planos y cartografías del estuario entre los años 1876 y 1932. Estos antecedentes permitieron tener una visión general del estado morfológico del estuario del río Maule.

Para la fotointerpretación se utilizaron nueve fotografías aéreas marca Aykon, 1: 70.000, del Servicio Aerofotogramétrico de la Fuerza Aérea (SAF 1:30.000) y el Servicio Hidrográfico y Oceanográfico de la Armada (SHOA), que cubren los años 1944-5 - 1997; e imágenes satelitales obtenidas del software Google Earth 7.1.2., correspondientes al período $2004-2011$ (Tabla 1). Existen al menos dos ejemplares para cada estación del año. 
TABla 1: Fotografías AÉREAS E IMÁGENES SATELITALES UTILIZADAS.

Table 1. Aerial Photographs and Satellite images USED

\begin{tabular}{|c|c|c|c|c|c|}
\hline $\mathbf{N}^{\circ}$ & $\begin{array}{c}\text { TIPO DE } \\
\text { MATERIAL }\end{array}$ & FUENTE & $\begin{array}{c}\text { FECHA DE } \\
\text { CAPTURA }\end{array}$ & AÑ & TEMPORADA \\
\hline 1 & Fotografía aérea & IGM & S/I & $1944 \sim 1945$ & Primavera-Verano \\
\hline 2 & Fotografía aérea & IGM & 18 de Enero & 1955 & Verano \\
\hline 3 & Fotografía aérea & IHN-MOP & Marzo & 1965 & Verano \\
\hline 4 & Fotografía aérea & IHA-SHOA & 26 de Noviembre & 1974 & Primavera \\
\hline 5 & Fotografía aérea & SAF & 11 de Agosto & 1978 & Invierno \\
\hline 6 & Fotografía aérea & IHN-MOP & S/I & 1979 & S/I \\
\hline 7 & Fotografía aérea & SAF & 7 de Abril & 1992 & Otoño \\
\hline 8 & Fotografía aérea & SAF & 3 de Octubre & 1994 & Primavera \\
\hline 9 & Fotografía aérea & SAF & 11 de Febrero & 1997 & Verano \\
\hline 10 & Imagen satelital & Google Earth 7.1.2 & 14 de Marzo & 2004 & Verano \\
\hline 11 & Imagen satelital & Google Earth 7.1.2 & 28 de Abril & 2007 & Otoño \\
\hline 12 & Imagen satelital & Google Earth 7.1.2 & 22 de Julio & 2009 & Invierno \\
13 & Imagen satelital & Google Earth 7.1.2 & 2 de Marzo & 2010 & Verano \\
14 & Imagen satelital & Google Earth 7.1.2 & 20 de Noviembre & 2011 & Primavera \\
\hline
\end{tabular}

El material gráfico fue digitalizado a través del software ArcGIS 10, resultando en un total de 14 esquemas morfológicos. Para facilitar la comparación entre dichos esquemas, se estableció la misma escada gráfica para todos ellos, y se incorporaron segmentos rectilíneos transversales al eje longitudinal del estuario, con el fin de evidenciar de mejor manera los avances o retrocesos experimentados por los individuos morfológicos constituyentes del estuario. Se midió también los principales bancos estuariales deltaicos, con el fin de demostrar los cambios que ellos han experimentado a través de los años.

El trabajo de terreno consistió en la caracterización de bancos estuariales y barras, según los criterios expuestos por ARAYA-VERGARA (1981).

Se consideraron datos correspondientes al caudal diario del estuario del río Maule, extraídos del sistema de estadística hidrológica en línea, perteneciente a la DIRECCIÓN GENERAL DE AGUAS (2012). Estos datos fueron revisados con el fin de encontrar cifras de gasto fluvial anómalo del río Maule, que pudiesen estar relacionados con cambios en la configuración morfológica estuarial, encontrando un caso significativo en el esquema del año 1997.

\section{RESULTADOS}

\section{Antecedentes cartográficos históricos de la morfología del estuario del Maule (1876 - 1932)}

Este análisis se hará en el siguiente orden, siguiendo la zonación de ARAYAVERGARA (1981), para sistema de deltas estuariales: delta estuarial, laguna estuarial y zona marina. En cada caso, los bordes del estuario se denominarán derecho e izquierdo, suponiendo un observador que mira hacia el mar.

En el Plano General del Puerto de Constitución del año 1876 (Fig. 4A), se puede observar que el delta estuarial se encuentra compuesto por dos bancos medios. El mayor de ellos se encuentra ubicado junto al borde izquierdo, y corresponde a un banco medio que en aquel tiempo era llamado La Isla, actualmente conocido como Isla Orrego. Este banco presenta una forma elíptica con extremos 
lanceolados, y una longitud total de más de $1 \mathrm{~km}$. El otro banco le sigue en tamaño, y se ubica muy próximo al borde derecho; posee una forma oblonga estimada en casi $1 \mathrm{~km}$ de longitud, por lo cual su presencia también es significativa al interior del estuario.

El plano también permite observar el estado de la laguna estuarial, delimitada por una flecha recurvada y bien desarrollada, proveniente del borde derecho.

El Plano del Litoral de Constitución del año 1905 (Fig. 4B) no posee un buen nivel de detalle gráfico, sin embargo su importancia radica en representar el delta estuarial; mantiene los bancos descritos en la Fig. 4A.

El esquema que muestra la superposición de planos históricos de los años 1876, 1891 y 1905 (Fig. 4C), presenta los cambios ocurridos en el delta estuarial y el sector de la flecha de Quivolgo, entre fines del siglo XIX y comienzos del XX. En él se puede ver la evolución morfológica del banco próximo al borde derecho (Fig. 4A). También se deduce que los bancos medios han aumentado de tamaño, aunque con tendencias de crecimiento contrarias, pues mientras el extremo distal de La Isla se ha dirigido preferentemente hacia la desembocadura, el extremo distal del otro banco medio ha retrocedido aumentando su superficie hacia aguas arriba, con respecto a su posición en 1876 . Si bien la representación de esta secuencia es algo confusa, a grandes rasgos, es posible reconocer un cambio en la zona de laguna estuarial, con reubicación algo más distal de la flecha litoral.

El aspecto más relevante que muestra el Plano del Puerto de Constitución del año 1932 (Fig. 4D), corresponde a la presencia de un banco medio, conformado por grava e identificado en condiciones de baja marea junto al borde izquierdo, hecho que se relacionará posteriormente con el proceso de crecimiento distal de Isla Orrego.
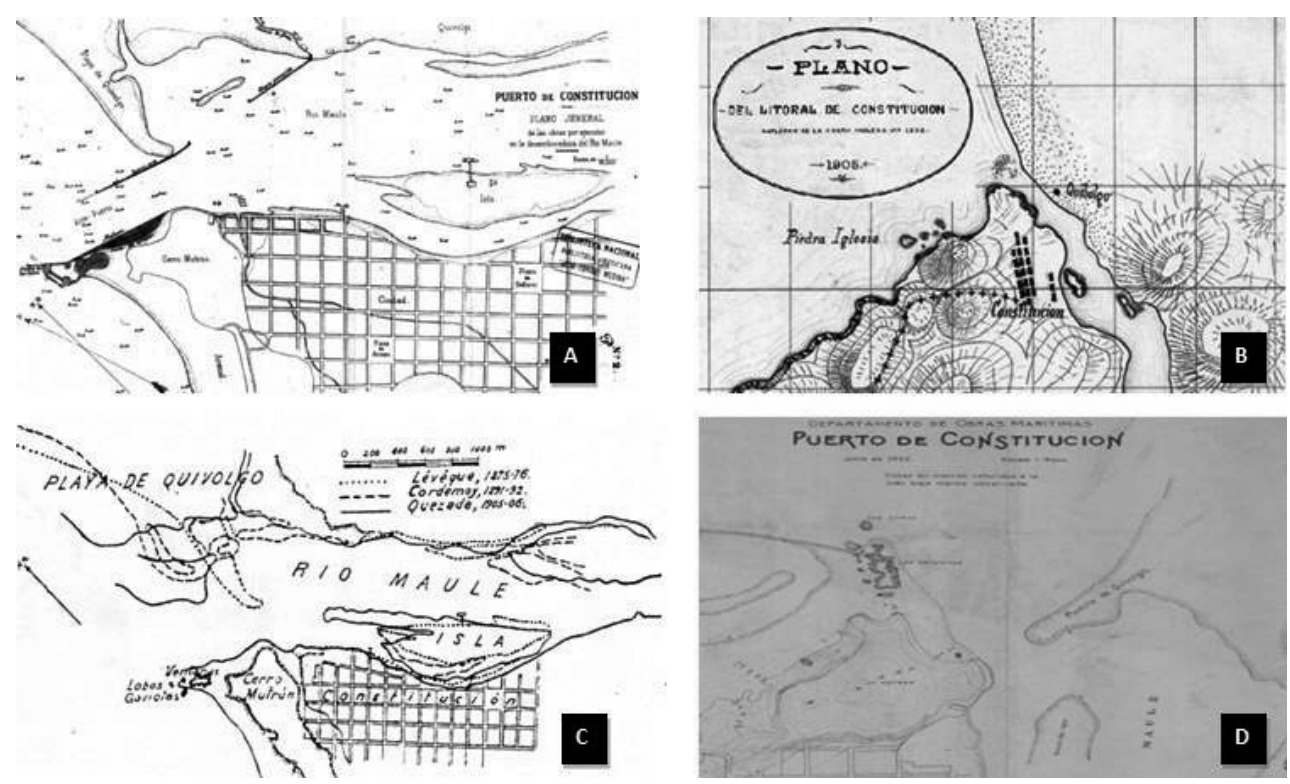

Fig. 4. Cartografías históricas del estuario del río Maule años: A) 1876, B) 1905, C) superposición de planos 1876, 1891 y 1905 y D) 1932.

Fig. 4. Hsitorical maps of Maule estuary years A) 1876, B) 1905, C) superposition of plans 1876, 1891 and 1905 and D) 1932. 
Análisis comparativo de los estados morfológicos entre 1944-5 y 2011

Así como en el caso de la revisión de mapas históricos, este análisis se hará en el siguiente orden, siguiendo la zonación de ARAYA-VERGARA (1981), para sistema de deltas estuariales: delta estuarial, laguna estuarial y zona marina. En cada caso, los bordes del estuario se denominarán derecho e izquierdo, suponiendo un observador que mira hacia el mar.

Estado morfológico años: 1944-5, 1955 y 1965 (Fig. 3)

\section{DELTA ESTUARIAL}

En 1944-5 (Fig. 5), la Isla Orrego está en la misma ubicación que presentó en el registro cartográfico histórico del período 1876-1905, aunque exhibiendo una mayor dimensión. Su forma es romboidal con extremos lanceolados, y presenta entrantes $\mathrm{o}$ irregularidades en ambos extremos. Al poniente de Isla Orrego, se identifican tres bancos medios irregulares, que presentan similar ubicación que el banco de ripio avistado en bajamar en la cartografía histórica del año 1932 (Fig. 4D). En1955, se ve un importante aumento de superficie en la Isla, sobre todo en el extremo distal. Producto de esta acreción, Isla Orrego adquiere una forma más alargada, llegando a alcanzar una longitud de casi $2 \mathrm{~km}$, lo que corresponde a la mayor longitud registrada dentro de la secuencia morfológica estudiada (Tabla 2). Además, es posible notar un aumento de entrantes, no sólo en los extremos sino que también en la parte media. En 1965, prácticamente se mantiene la forma y dimensión que Isla Orrego exhibía en 1955, con excepción de la entrante antes presentada en el extremo proximal, que al parecer terminó por desaparecer provocando una reducción de su extensión total, y a su vez, dando origen a otro banco medio.

En 1944-5, el banco medio Isla Del Perro se encuentra ubicado muy próximo al borde derecho del estuario, separado de ella sólo por un estrecho canal, posición similar a la que presentaba en el año 1905 en la superposición de planos históricos. Este banco presenta una forma cuasi-romboidal con extremos lanceolados y numerosas entrantes, la principal de ellas ubicada al en el extremo proximal. En 1955 el banco ha cambiado su forma robusta a una más alargada, con dos importantes entrantes dispuestas en sentido contrario una con la otra. Por otro lado, el canal que aún independiza a este banco del borde derecho, se ha tornado mucho más estrecho aguas arriba. En1965 el banco no presenta cambios aparentes. Sin embargo, en el lapso entre 1944 y 1965 aparece un banco lateral separado del banco El Perro por un canal, rasgo que se agrega a la configuración del delta estuarial (Fig. 5)

TABla 2: VARIABILIDAd EN LAS DIMENSIONES de LOS INDIVIDUOS MORFOLÓGICOS DELTAICOS DEL estuario del Maule, medido entre los años 1944 y 2011 (Medido en metros).

TAble 2. Variations of The dimensions of the deltaic MORPhologies in the MaUle estuary MEASURED BETWEEN 1944 AND 2011 (METERS).

\begin{tabular}{|c|c|c|c|c|c|c|c|c|c|c|c|c|c|c|c|c|}
\hline \multicolumn{2}{|c|}{ PRINCIPALES BANCOS DELTAICOS } & DIMENSIONES & $1944-5$ & 1955 & 1965 & 1974 & 1978(a) & 1978(b) & 1992 & 1994 & 1997 & 2004 & 2007 & 2009 & 2010 & 2011 \\
\hline \multirow{2}{*}{\multicolumn{2}{|c|}{ Isla Orrego }} & Longitud & 1334 & 1904 & 1634 & 1369 & 1311 & 1292 & 1083 & 1102 & 1043 & 1092 & 1110 & 1088 & 1020 & 1082 \\
\hline & & Ancho & 364 & 318 & 321 & 290 & 283 & 269 & 212 & 239 & 250 & 212 & 198 & 198 & 196 & 217 \\
\hline \multirow{2}{*}{\multicolumn{2}{|c|}{ Banco del Edén }} & Longitud & 1590 & 1721 & 1734 & 1384 & 1351 & 1339 & 1312 & 1339 & 1324 & 1314 & 1304 & 1288 & 1280 & 1237 \\
\hline & & Ancho & 524 & 520 & 521 & 534 & 544 & 513 & 345 & 372 & 441 & 412 & 398 & 393 & 387 & 413 \\
\hline \multirow{6}{*}{$\begin{array}{l}\text { Isla del } \\
\text { Perro }\left({ }^{*}\right)\end{array}$} & \multirow{2}{*}{ Un banco medio } & Longitud & & & & & 483 & 555 & & 1188 & & 986 & & & & 970 \\
\hline & & Ancho & & & & & 129 & 150 & & 171 & & 156 & & & & 147 \\
\hline & \multirow{4}{*}{$\begin{array}{l}\text { Compuesta por dos } \\
\text { bancos medios }\end{array}$} & Longitud & & & & 530 & & & 645 & 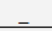 & 707 & - & 674 & 669 & 667 & \\
\hline & & Ancho & & & & 140 & _ & 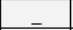 & 74 & - & 126 & - & 127 & 119 & 120 & \\
\hline & & Longitud & - & - & - & 170 & - & _ & 399 & - & 449 & - & 445 & 447 & 448 & \\
\hline & & Ancho & & & & 67 & & & 70 & & 94 & & 113 & 96 & 101 & \\
\hline
\end{tabular}

(*) Dada su variación morfológica, la Isla del Perro puede presentar uno o dos bancos medios. Elaborado según mediciones de los esquemas morfológicos 


\section{LAGUNA ESTUARIAL}

En la zona distal del estuario, la configuración morfológica de 1944-5 muestra una flecha litoral que nace desde la playa desde el borde derecho y se proyecta en dirección transversal al estuario. La flecha es más bien estrecha, corta y recurvada. En 1955, es más robusta que en el año anterior y con una particular forma algo triangular, interpretada como un abanico de derrame en la post playa. Esta forma también es identificada en el registro cartográfico histórico de 1876, la que puede ser entendida como relicto, que no alcanzó a ser afectado por el abanico de derrame. En 1965 ésta presenta un aspecto aún más imponente que en los esquemas anteriores, pues, además de haber estado afecta a un abanico de derrame, parece presentar una reactivación de su crecimiento distal. El desarrollo alcanzado en este año, denota condiciones hidrodinámicas favorables para su desarrollo.

\section{ZONA MARINA}

En la zona distal del estuario del Maule, se observa también la presencia de un sistema de barras frente a la desembocadura. En 1944-5, se ve un conjunto de dos a tres barras, una exterior discontinua y dos interiores con segmentación lateral. Entre la exterior y las interiores, hay un surco inter barras que sigue la dirección general del estuario. En 1955, se observa casi desaparición del sistema externo y una sola barra discontinua interna . En 1965 desaparece la estructura en dos sistema de barra y aparece una sola barra que atraviesa casi en su totalidad la boca del estuario, como una barra submarina simple y continua.

Estados morfológicos en los años 1974, 1978 y 1979 (Fig. 6)

\section{DELTA ESTUARIAL}

En 1974, Isla Orrego ha reducido su tamaño con respecto a 1965 . Si bien, la pérdida de superficie fue experimentada de manera general en todo el contorno, es más evidente en los extremos, siendo el extremo proximal el que ha experimentado un mayor retroceso. En este caso, la pérdida de superficie es más bien un desmembramiento del banco. En 1978 y 1979, aún persiste la forma y dimensión de este banco con respecto al año 1974, pero el en 1978 la Isla es más robusta.

En 1974, el banco del Perro experimentó un significativo desmembramiento desde el extremo proximal (Fig. 6 y Tabla 2). Éste hecho se asocia con la entrante de mayor importancia vista durante los años anteriores, la cual probablemente terminó por ceder generando un nuevo integrante del delta estuarial. Se trata de un pequeño islote estuario arriba del banco del Perro. Tras la segmentación, este banco adoptó una nueva forma, cóncava en el sector proximal y convexa en el distal. Las configuraciones morfológicas de los años 1978 y 1979 expresan un progresivo retroceso del borde medio-distal de este banco. Por otro lado, cabe señalar que, junto al banco medio El Perro, es clara la presencia de un banco lateral, levemente separado de su borde proximal.

El islote generado partir del sector proximal del banco Del Perro según esquema de 1974, está compuesto de dos bancos medios de forma irregular. En 1978 conformaba sólo un banco medio, pero en 1979 vuelve a estar constituido por dos bancos.

\section{LAGUNA ESTUARIAL}

En 1974, el límite marino de la laguna estuarial es una flecha estrecha, relativamente corta y con punta recurvada, proveniente del borde derecho. En 1978, la flecha es poco desarrollada, pero más robusta, pudiendo ser una flecha embrionaria o el relicto de una flecha removida. Por último, en 1979 no hay flecha. Se debe mencionar que el escaso o nulo desarrollo de estas formas se puede deber a que fueron captadas en fotografías aéreas tomadas en invierno y primavera, reflejando el régimen pluvio-nival del río Maule. Además, se debe considerar que 


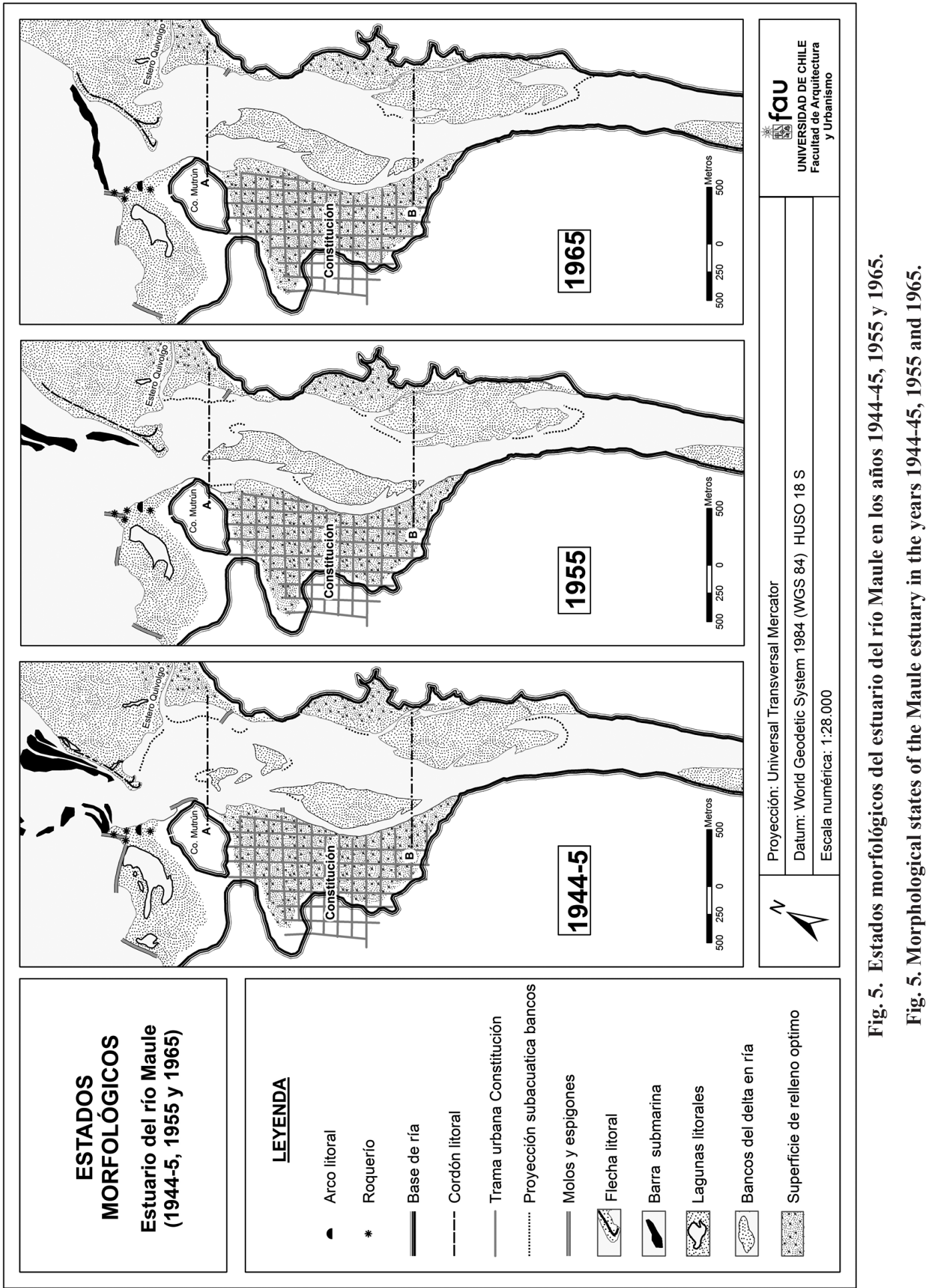


en invierno los estados del mar pueden ser desfavorables para el crecimiento de flechas.

\section{ZONA MARINA}

En 1974 las barras frente a la boca estuarial son segmentadas. Se trata de un conjunto de al menos 4 barras consecutivas, dispuestas de manera oblicua frente a la desembocadura y una barra aislada proyectada desde la incipiente flecha, que converge con el complejo de barras. En 1978 se observa una barra recurvada compleja y discontinua. En el promontorio sur junto a la boca las barras divergen hacia el mar, acompañadas de elementos aislados. En 1979, también se presenta una barra recurvada compleja y discontinua, aunque esta vez acompañada por un conjunto de barras lateralmente segmentadas ubicadas frente a la desembocadura. Se debe señalar que la configuración de barras recurvadas presentes en los esquemas de 1978 y 1979 parece estar asociada con crecidas del río Maule.

Estados morfológicos en los años 1992, 1994 y 1997 (Fig. 7 y Tabla 2)

\section{DELTA ESTUARIAL}

En 1992, se aprecia disminución de la superficie de Isla Orrego en relación con estado de 1979. No obstante, se debe señalar que entre ambas imágenes existe un período de tiempo de más de una década. La mayor pérdida de superficie parece haber sido focalizada en su extremo proximal, tal como se ha observado en años anteriores. Inmediatamente aguas arriba de Isla Orrego, se identifica un pequeño banco medio, heredero de fragmentación anterior. En 1994, se observa que Isla Orrego es de similar forma y disposición que el año anterior, aunque algo más robusto, con un leve crecimiento del extremo distal, pero también con poco retroceso del extremo opuesto. El pequeño banco medio ubicado inmediatamente aguas arriba, ha aumentado levemente su tamaño. En 1997 Isla Orrego ha mantenido su superficie con respecto a 1994, sin embargo, su extremo distal ha retrocedido ligeramente. El pequeño banco medio posicionado inmediatamente al oriente, ha reducido notablemente su superficie respecto a 1994. La construcción de un espigón en el extremo proximal de la Isla, la ha protegido de la erosión por desmembramiento a la que ha estado afecta Isla Orrego desde 1965.

El segundo cambio más importante corresponde al banco Del Perro. Después de la segmentación de su extremo proximal en 1974, y corresponde a una reducción de superficie, en el extremo medio-distal, observado en 1992. La nueva forma adoptada por el banco Del Perro, se mantendrá prácticamente igual en los siguientes años; en 1994 y 1997 hay conservación de forma.

En 1992, el producto de segmentación proximal del banco Del Perro se ve conformado por tres bancos, notablemente estrechos y alargados con respecto a los años anteriores. En 1994, aparece sólo un banco de gran longitud y superficie. En 1997 está seccionado en dos bancos medios, mostrando una morfología muy semejante a la anterior, aunque con una superficie más reducida. También existe un pequeño banco que no había sido identificado en los años anteriores, ubicado cerca del extremo distal del banco Del Perro.

\section{LAGUNA ESTUARIAL}

En 1992 se observa una flecha resguardada por el promontorio del borde izquierdo. Puede considerarse como flecha asociada a la laguna estuarial. Su disposición es más bien oblicua, y su punta se proyecta hacia Isla Orrego. Cerrando la laguna estuarial, se observa también que se ha formado la flecha procedente del borde derecho, alargada, estrecha y recurvada. En1994, la flecha asociada a la laguna estuarial alcanza un nivel de crecimiento superior al visto anteriormente, muy robusta en el sector desde el cual se encuentra adosada a la ribera. Se deduce que las condiciones 


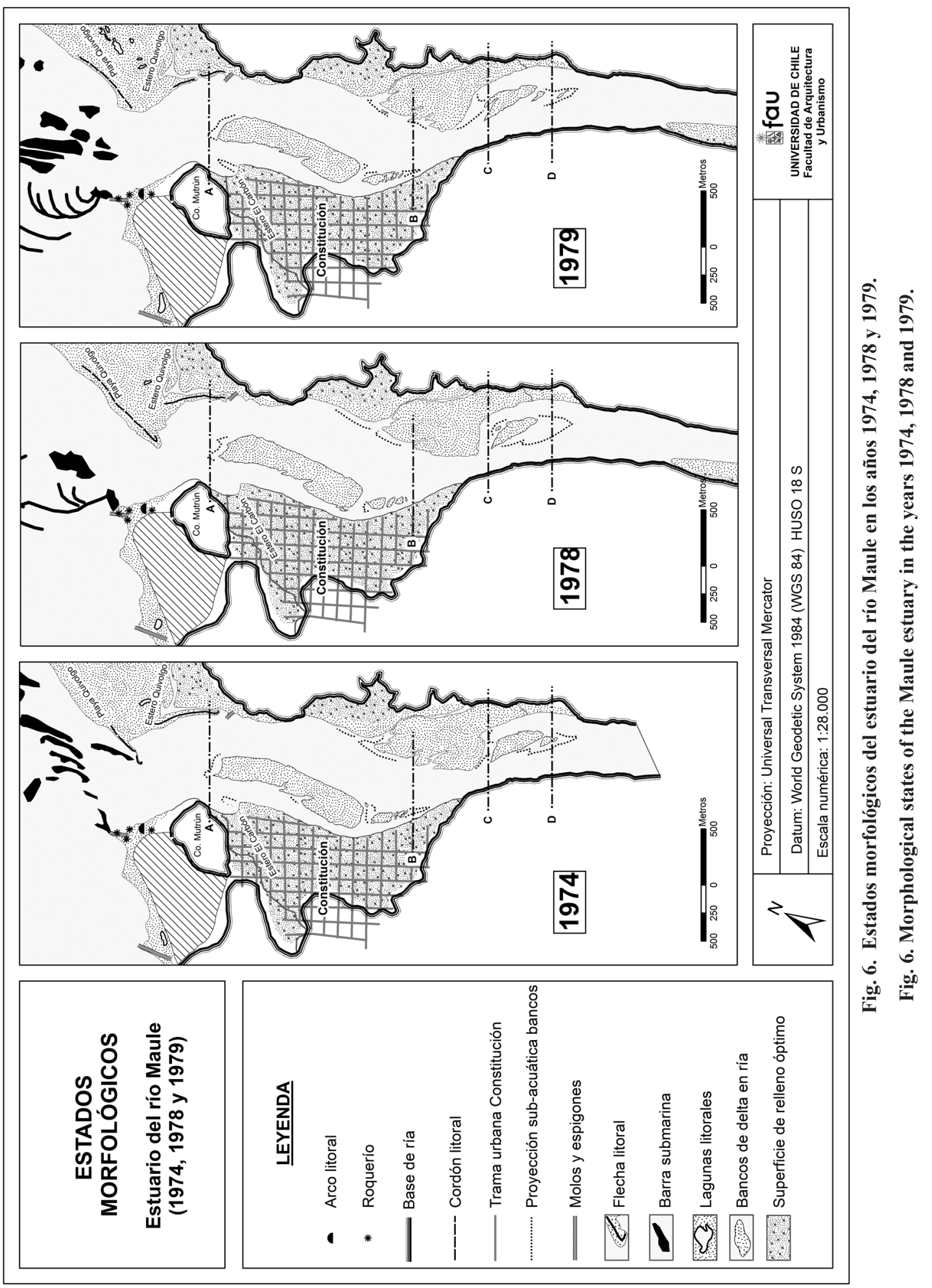


que generaron la flecha en 1992, se han repetido en 1994: Inversamente, falta la flecha del borde derecho. En 1997 el cierre externo de la laguna estuarial presenta dos flechas, como en 1992, pero más desarrolladas. La presencia y desarrollo de ellas han sido suficientes para reducir el ancho de la boca del Maule a 90 m, este hecho puede relacionarse de manera directa con la notable baja de caudal sufrida en aquella fecha (Tabla 3). Una flecha exterior, asociada al promontorio sur que delimita la boca del estuario, debe su origen a la deriva litoral.

\section{ZONA MARINA}

En 1992, la zona marina presenta una barra simple curvada hacia el mar, acompañada de barras aisladas. En 1994, se aprecia una barra discontinua segmentada lateralmente, y en 1997 una barra simple discontinua.

Tabla 3. Caudal del río Maule registrado en la estación fluviométrica de Forel, COINCIDENTE CON LA FECHA DE CAPTURA DE LAS FOTOGRAFÍAS E IMÁGENES BASE DE LOS ESQUEMAS MORFOLÓGICOS DE LOS AÑos 1992, 1994 y 1997.

Table 3. Flow of the Maule River registered in the Forel fluviometric station, coinciding WITH THE DATE OF THE PHOTOS AND IMAGES OF MAPS IN THE YEARS 1992, 1994 AND 1997.

\begin{tabular}{|c|c|c|c|c|}
\hline \multirow{2}{*}{$\begin{array}{c}\text { FECHA FOTOGRAFíA } \\
\text { AÉREA O IMAGEN } \\
\text { SATELITAL }\end{array}$} & ESTACIÓN & \multicolumn{3}{|c|}{ CAUDAL $\left(\mathrm{m}^{3} / \mathrm{s}\right)$} \\
\cline { 3 - 5 } & DEL AÑO & $\begin{array}{c}\text { Caudal promedio } \\
\text { día }\end{array}$ & $\begin{array}{c}\text { Caudal promedio } \\
\text { mes anterior }\end{array}$ & $\begin{array}{c}\text { Caudal promedio } \\
\text { año anterior }\end{array}$ \\
\hline 7 de Abril 1992 & Otoño & 224 & 274 & 390 \\
\hline 3 de Octubre 1994 & Primavera & 479 & 417 & 462 \\
\hline 11 de Febrero 1997 & Verano & 67 & 70 & 246 \\
\hline
\end{tabular}

Fuente: Elaboración propia, a partir de datos extraídos de la Dirección General de Aguas, (DGA 2012). Source: Based on data from the Dirección General de Aguas, (DGA 2012).

Estados morfológicos en los años 2004 y 2007 (Fig. 8)

\section{DELTA ESTUARIAL}

En 2004, el delta estuarial presenta a Isla Orrego conservando en gran medida su forma y superficie con respecto a 1997. No se registra un retroceso del extremo proximal como ha ocurrido en años anteriores, debido a la protección del molo instalado en dicho sector. El banco medio posicionado inmediatamente aguas arriba de Isla Orrego, es alargado y estrecho. En 2007, Isla Orrego posee la misma forma que en la fecha anterior. En esta dos fechas, no se ve cambios significativos del banco Del Perro, salvo que el borde distal de éste ha retrocedido levemente debido al despojo de las estructuras de muelles antes instaladas allí. Los bancos distales cercenados de este banco no presentan grandes cambios. No obstante, en 2004, estos bancos se presentan unidos, aunque en 2007 nuevamente aparecen separados.

\section{LAGUNA ESTUARIAL}

En 2004, el cierre de la laguna está indicado por dos flechas, a ambos lados de la boca estuarial. La flecha izquierda, posee una proyección curvada hacia el mar, siguiendo la orientación de la barra submarina, indicando que se proyectya en ésta. La flecha derecha presenta una apariencia muy robusta, pero de escaso desarrollo longitudinal. En 2007, hay ausencia de flechas, lo que posiblemente está asociado a la dinámica estacional. 


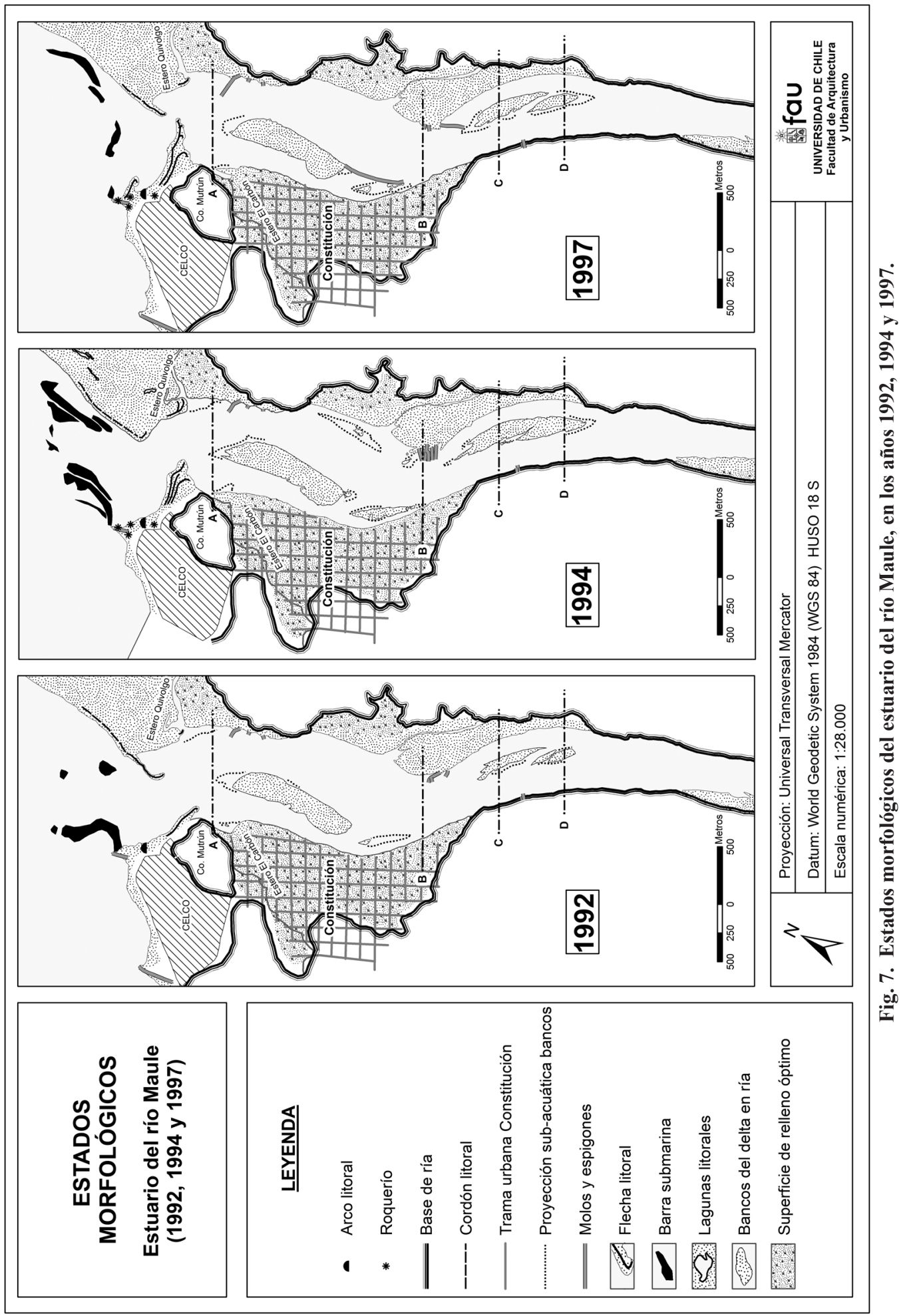




\section{ZONA MARINA}

En 2004, se ve una barra discontinua, segmentada lateralmente. Su extremo sur deriva de la flecha externa sur. Su trayectoria es aproximadamente paralela a la línea litoral de Quivolgo. En 2007, es posible observar la existencia de una barra discontinua derivada del extremo norte de la desembocadura, dispuesta de manera oblicua a la playa de Quivolgo.

Estados morfológicos de los años 2009, 2010 y 2011

\section{DELTA ESTUARIAL}

El esquema del año 2010, corresponde al estado del estuario del Maule tres días después de ocurrido el tsunami de 27.02.2010 e incorpora el área de influencia en las zonas ribereñas del estuario.

En 2009, Isla Orrego tiene un aspecto muy similar al esquema del año 2007, pero con su extremo distal algo disminuido. En 2010 pese al embate de las olas tsunámicas de 2010, Isla Orrego no sufrió grandes cambios con respecto a su superficie y forma (Fig. 9 y Tabla 2). No obstante, como efectos asociados se hallan una leve erosión en los bordes del extremo proximal, un aumento de entrante principal e inundación parcial del extremo distal por lagunillas. Cabe señalar que es muy probable que Isla Orrego haya sufrido erosión sobre su superficie, sin embargo esta información no fue cubierta. Por otro lado, el espigón proximal resistió el tsunami, siendo poco abatido cerca de la isla. En 2011, Isla Orrego ganó superficie con respecto al año anterior, mostrándose más robusta, aunque conservando siempre su forma.

La morfología de los años 2009, 2010 y 2011 presentan al banco Del Perro en similares condiciones que en el año 2007. No obstante, en el año 2010 éste se presenta un tanto más disminuido en el extremo distal, y cuenta con una lagunilla interior probablemente dejada por paso del tsunami. Los pequeños bancos proximales cercenados de este banco son dos en 2009 y 2010, mientras que en 2011 ello se observan unidos en uno solo.

\section{LAGUNA ESTUARIAL}

En 2009 una robusta flecha de escaso desarrollo longitudinal está adosada al margen norte de la boca del estuario. En 2010, esta forma no existe debido a que fue destruida por el tsunami de este año. El retroceso sufrido por la línea litoral en este sector, fue mucho más severo que el presentado en esquemas anteriores analizados a través de fotografías de estación invernal. Además, grandes lagunas fueron dejadas en la playa correspondiente. Por el contrario, en el año 2011 se aprecia una robusta e imponente flecha de Quivolgo. Este aumento de superficie responde a la reacomodación de la gran cantidad de material sedimentario que quedó disponible frente a la costa luego de un tsunami.

\section{ZONA MARINA}

En 2009, se ve rompientes de barras aisladas, oblicuas frente a la playa. En 2010, se aprecia una barra simple discontinua algo oblícua a la playa. En 2011, aparece una barra simple discontinua, sensiblemente perpendicular a la boca del estuario.

\section{DISCUSIÓN}

\section{Morfología del delta estuarial}

\section{Canales estuariales}

Durante el período observado, se observa siempre un canal principal ancho y dos canales laterales angostos. Luego, ambos rasgos son formas persistentes. El canal principal describe meandros de baja curvatura. Los canales laterales separan los bancos deltaicos principales del borde de la ría. 


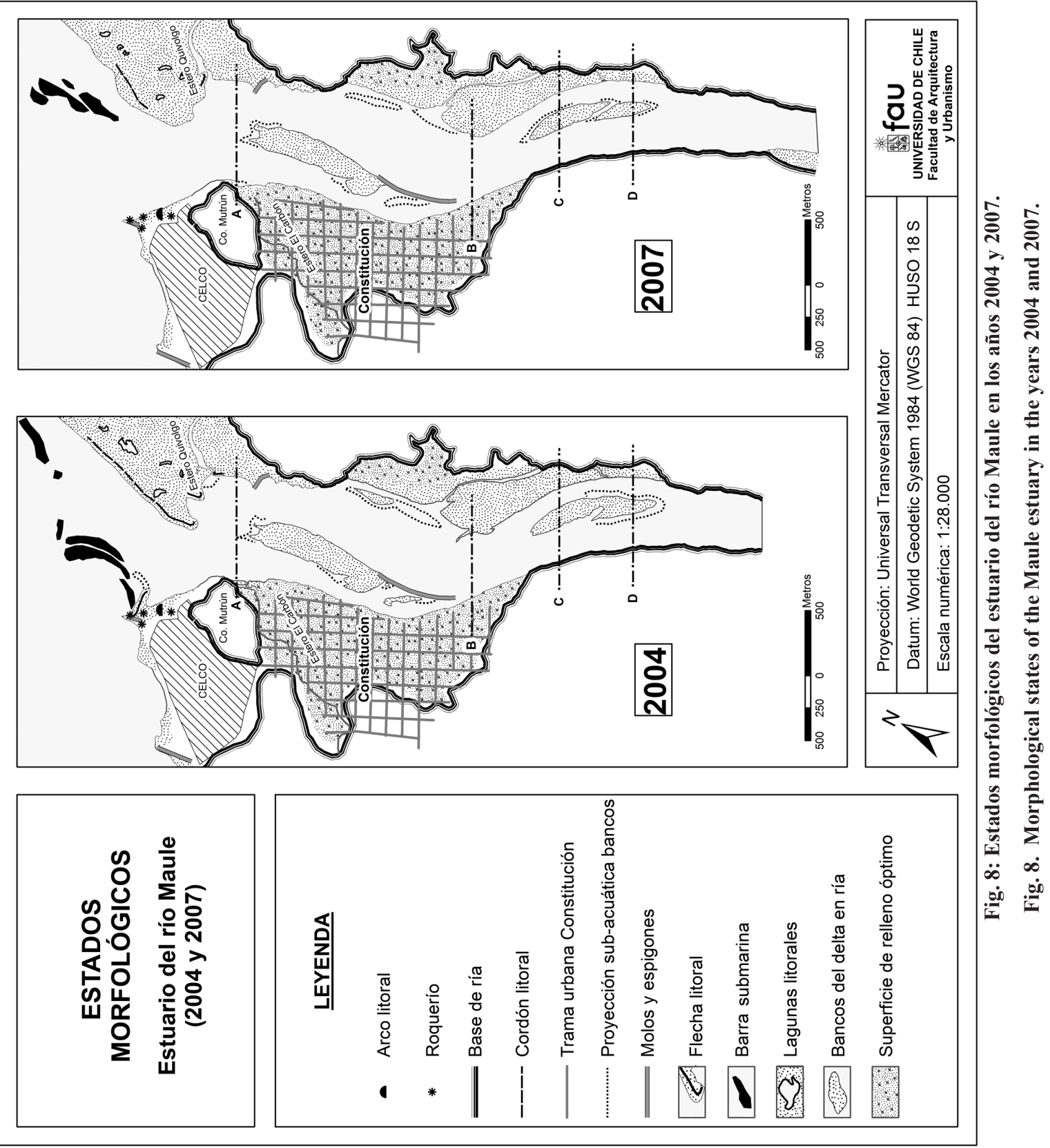




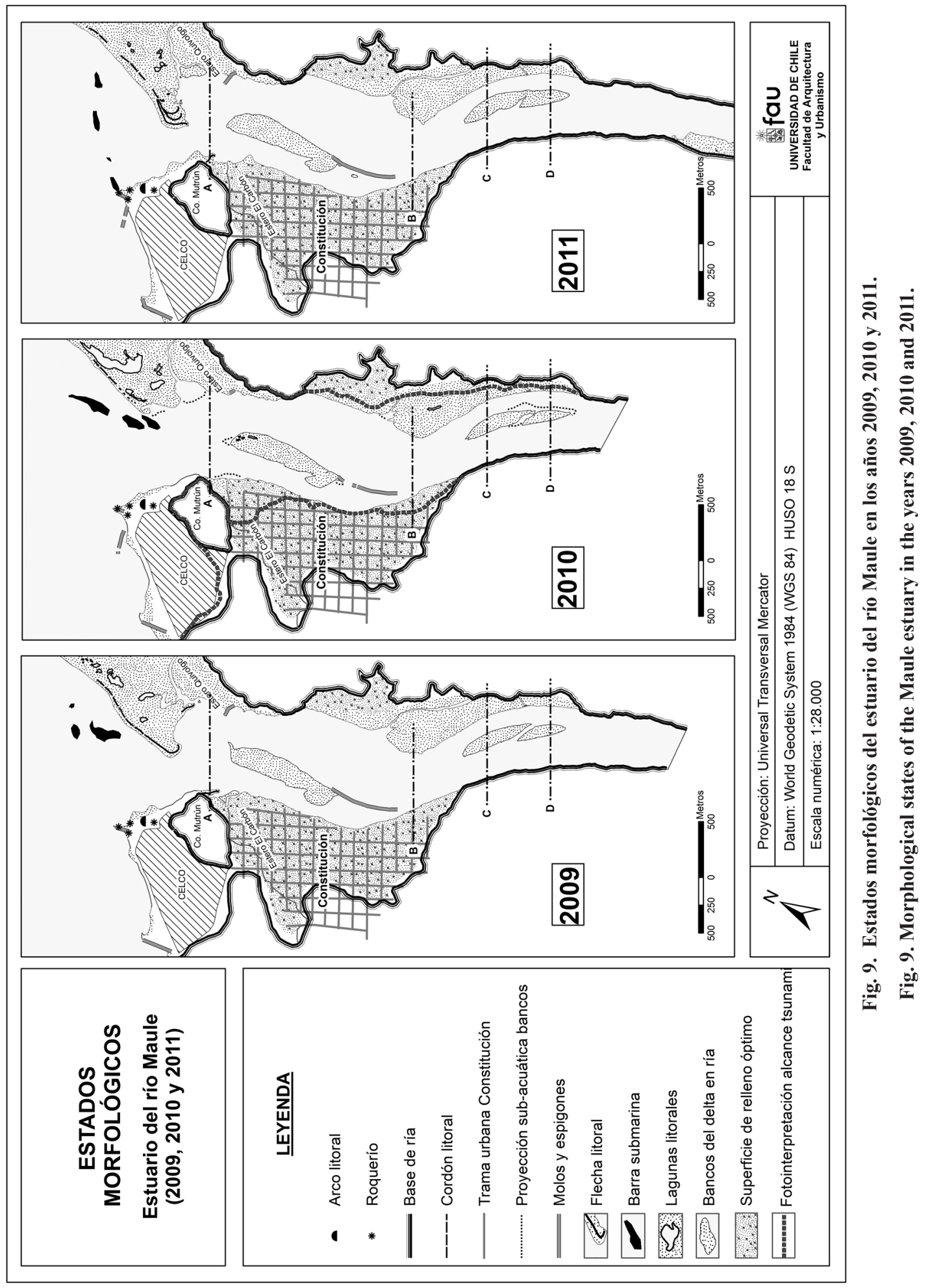


Bancos deltaicos medios persistentes

Estos bancos son alargados y siguen la misma dirección que los bordes del canal principal. Temporalmente, pueden sufrir segmentaciones impersistentes. El análisis de ARAYA-VARGARA (1981) indica que el proceso formador de las formas persistentes consistió en acreción de bancos menores.

\section{Bancos laterales}

Su grado de persistencia no es claro. Están separados de los bancos medios por los correspondientes canales laterales.

\section{Morfología comparada}

Esta estructura morfológica es excepcional en Chile Central (ARAYA-VERGARA 1981), donde lo común en los principales sistemas es la anastomosis entre canales y bancos deltaicos, hecho que no se advierte en el caso del Maule. Diferentes casos analizados en el mundo indican variabilidad espacial y temporal en los deltas estuariales. En Sudáfrica, COOPER (1993 y 1994) encontró notables cambios de patrón anastomosado de bancos y canales en aproximadamente 50 años (1931-1983), conduciendo -finalmente- a acreción de bancos. Registró, en seguida, cambios drásticos en 3 años (1987-1990), producidos por crecidas fluviales. Se documentó, así, el paso de un sistema anastomosado a uno de canal principal con bancos acrecionados. También COOPER (2001), para estuarios micromareales de costa sudafricana con oleaje dominante, halló bastante variedad entre anastomosis y canal unificado, dependiendo ella del grado de influencia mareal.

Rasgos específicos han sido encontrados en el SW de India. AVINASH et al. (2012) hallaron impactos evolutivos en la relación entre bancos anastomosados y canal principal: En el estuario Swarna, identificaron dos dominios referentes a los bordes de la ría: el de los bancos anastomosados junto al borde izquierdo y el del canal principal, junto al borde derecho. Por su parte, observaciones para un lapso de 100 años en el estuario del Yangtze (China) indican poco cambio en los rasgos fundamentales de un delta semianastomosado con canales inter bancos. Éstos se presentan como distributarios parecidos a los de un delta marino (HE et al. 2013; ZHANG et al. 2015). Esta estructura no se encuentra en los demás casos relatados.

\section{Factores}

De lo expuesto como geomorfología comparada y de la discusión específica de COOPER (1993, 1994 y 2001) que considera los casos chilenos (en ARAYA-VERGARA 1985), se deduce que éstos están influidos por cuencas desarrolladas en un país de fuerte pendiente $y$ de precipitaciones suficientes. Se desprende que la ubicación de los casos chilenos como dependientes del dominio fluvial en el triángulo de clasificación de COOPER (1993 y 1994) debe ser discutida. La revisión de ARAYAVERGARA (1985) hecha por COOPER (1993) le inducen a tener en cuenta que es importante considerar en la costa chilena su exposición a un régimen de alta energía de oleaje, como se verá en seguida.

\section{Morfología de laguna estuarial y zona marina}

Si se compara el grado es estabilidad del delta estuarial con el de las flechas y barras asociadas a la laguna estuarial y la zona marina, se advierte que estas últimas son realmente impersistentes. Esta impersistencia es común en Chile Central e indica frecuente cambio en la energía del oleaje y en el grado de importancia de la deriva litoral. Esto ya ha sido discutido principalmente por ARAYA-VERGARA (1985) y COOPER (1993 y 2001). 
Importancia de los sedimentos en la morfología general del sistema

Además de la reconocida intensidad de los parámetros hidráulicos ya especificados para la morfodinámica del sistema, es necesario considerar el tamaño de grano de los sedimentos aportados al estuario. Observaciones de ORTON \& READING (1993) indican las posibles influencias de este factor en la morfogénesis y el grado de persistencia de las formas. Por lo tanto, estos autores enfatizan la necesidad de investigación sobre este tema. En el caso del Maule, algunas mediciones sobre morfometía y litología de gravas del banco estuarial Isla Orrego inducen a establecer las posibles cuencas de origen de estos materiales (ARAYA-VERGARA 1981), pero este conocimiento es insuficiente e incita a futuras investigaciones.

\section{CONCLUSIONES}

La generalizada persistencia de los bancos del delta estuarial, sin cambios notables de forma y de tamaño, indica que el balance entre erosión y progradación fluvial opera bajo un régimen de retroalimentación negativa, sin cambios fundamentales en el régimen fluvial. En este sentido, se puede admitir que esta forma está controlada por un mecanismo de estabilidad fluvial, que regula un delta estuarial de dominio fluvial. En contraste, la impersistencia de las formas relacionadas con la laguna estuarial y la zona marina induce a concluir que estas formas son dominadas por oleaje de alta energía. Desde el punto de vista morfodinámico, las comparaciones entre el caso del Maule y otros similares deben hacerse separando las zonas de delta estuarial, laguna estuarial y marina.

\section{REFERENCIAS}

ARAYA-VERGARA, J.F., 1970. Contribución al estudio de los procesos estuariales en las desembocaduras de los ríos Rapel y Maipo. Inform. Geogr., Chile, 20: 17-38.

ARAYA-VERGARA， J.F., 1981. El concepto de delta en ría y su significado en la evolución litoral (ejemplo de Chile Central). Inform. Geogr., Chile, 28: 19-42.

ARAYA-VERGARA, J.F., 1982. Análisis de la localización y de los procesos y formas predominantes de la línea litoral de Chile. Inform. Geogr., Chile, 29: 35-55.

ARAYA-VERGARA, J.F., 1985. Sediment supply and morphogenetic response on a high wave energy coast. Z. Geomorph. N.F.S.B. 57: 67-79.

ARAYA-VERGARA, J.F., 2007. Ocean Coasts and Continental Shelves. In: Veblen, Th.T., K.R. Young \& A.R. Orme (Eds.), The Physical Geography of South America. Oxford Univ. Press, N. York. Chap. 15: pp. 249-261.

ARRIAGADA, J., 2005. Cambios en el sistema estuarial del Maipo y su relación con obras portuarias, Chile central. Memoria para optar al título de geógrafo, Universidad de Chile, Santiago; 80 pp. Inédito.

AVINASH, K., K.S. JAYAPPA \& P. VETHAMONY, 2012. Evolution of Swarna estuary and its impact on braided islands and estuarine banks, Southwest coast of India. Environ Earth Sci., 65: 835-848.

COOPER, J.A.G., 1993. Sedimentation in a river dominated estuary. Sedimentology, 40: 979-1017. 
COOPER, J.A.G., 1994. Sedimentary processes in the river-dominated Mvoti estuary, South Africa. Geomorphology, 9: 271-300.

COOPER,J.A.G.,2001.Geomorphological variability among microtidal estuaries from the wave-dimunated South African Coast. Geomorphology, 40: 99-122.

CORDEMOY, C., 1893. Estudio relativo a los puertos de Constitución y Corral y las mejoras de los ríos Maule y Valdivia. Imprenta Nacional, Santiago, 224 pp.

CORTÉZ, C., 2002. Observaciones dinámicas y geomorfológicas en el estuario del Aconcagua, Chile Central. Memoria para optar al título de geógrafo, Universidad de Chile, Santiago; 73 pp. Inédito.

DAVIES, J.L., 1977. Geographical Variation in Coastal Development. Longman, London.

DAVIES, J.L., 1982. World net sediment transport: In: Schwartz, M.L. (Ed.), Encyclopedia of Beaches and Coastal Environments. Hutchinson Ross, Stroudsburg, pp. 881-882.

DIRECCIÓN DE OBRAS PORTUARIAS \& LABORATOIRE CENTRALE D'HYDRAULIQUE DE FRANCE, 1960-1. Misión hidrográfica Puerto de Constitución. I: Informe General (Inédito).

DIRECCIÓN GENERAL DE AGUAS, 2012. Caudal medio diario: Río Maule en estación Forel 1992 - 2011. Centro de Información de Recursos Hídricos. Dirección General de Aguas (DGA). Ministerio de obras públicas (MOP). Santiago, Chile.
HE, Y., CHENG, H. \& CHEN, J., 2013. Morphological evolution of mouth bars on the Yangtze estuarine waterways in the last 100 years. J. Geogr. Sci., 23(2): 219-230.

LEVEQUE, A., 1876. Estudio sobre la ría de Constitución i la barra del río Maule: Memoria Técnica i anexo presentado al señor Ministro de Marina. Librería del Mersurio, Santiago. 97 pp.

MARTINEZ C. \& C. CORTEZ, 2008. Características geomorfológicas del estuario Aconcagua, Chile Central., entre 1877 y 2001. R. Geogr. Valpso., 41: 14-26.

MIKHAILOV, V.N. \& S.L. GORIN, 2012. New Definitions, Regionalization, and Typification of River Mouth Areas and Estuaries as Their Parts. Water resources, 39(3): 247-260.

ORTON, G.J. \& H.G. READING, 1993. Variability of deltaic processes in terms of sediment supply, with particular emphasis on grain size. Sedimentology, 40: 475-512.

POMAR, J., 1963. Cambios en los ríos y en la morfología de la costa de Chile. R. Chil. de Hist. y Geogr., 130: 318-356.

ZHANG, L., B. WU, K. YIN, X. LI, K. KIA \& L. ZHU, 2015. Impact of human activities on the evolution of estuarine wetland in the Yangtze Delta, from 200 to 2010. Environ Earth Sci., 73: 435-447. 

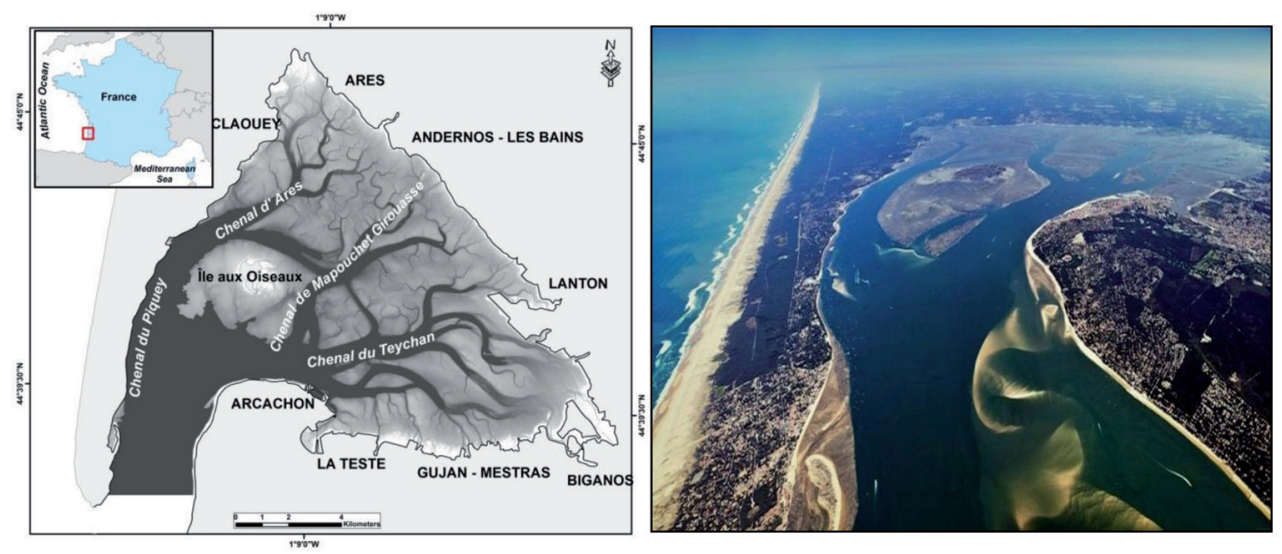

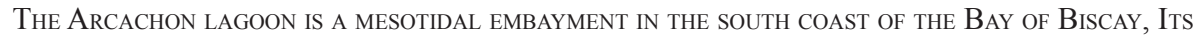
TOTAL SURFACE IS ABOUT $174 \mathrm{KM}^{2}$ AT HIGH TIDE, WHERE 65\% IS FORMED OF TIDAL FLATS. FROM THE PERIOD OF THE OLDEST RELIABLE BATHYMETRIC MAP (1865), THE SEDIMENT BUDGET ESTIMATED FOR 126 YEARS SHOWED SEDIMENTARY ACCRETION WITHIN TIDAL FLATS WHICH RARELY EXCEEDED $+0.5 \mathrm{M}$, WHEREAS THE MAIN DISTRIBUTARY CHANNELS CONNECTED TO THE TIDAL INLET EXPERIENCED NET EROSION OF A FEW METERS. AT PRESENT, THE MOST RECENT OBSERVATIONS MADE BY END-USERS OF THE LAGOON, SUGGEST RELATIVE INFILLING OF THE CHANNELS (AlLARD ET AL., 2009) AND A RELATIVE INCREASE OF TURBIDITY IN THE INNER WATERS. ThiS SUGGESTS THAT A MOBILE STOCK OF SURFICIAL FINE SEDIMENT IS AVAILABLE IN THE LAGOON, WHICH CAN BE EXCHANGED BETWEEN THE TIDAL FLATS AND THE CHANNELS, DEPENDING ON THE HYDRODYNAMIC CONDITIONS. (Departamento de Geografîa/Doctorante-Physique de l'Environnement, Université Bordeaux, Francia). Joselyn ArRiagada. JOARRIAG@UChILE.CL

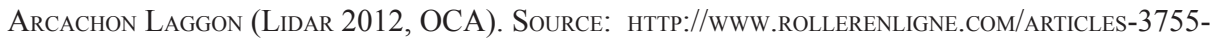
RANDO-VERTE-ROLLER-2015-LA-BOUCLE-DU-BASSIN-D-ARCACHON-33.HTML 